collaborators. Studying this faint population will yield a more complete picture of their role in reionizing the Universe. The authors' preliminary foray in studying the first galaxies underscores the important role of facilities such as the JWST in revolutionizing our understanding of galaxy formation at the earliest cosmic epochs, and paves the way for a bright future in studying faint and distant galaxies.
Naveen A. Reddy is at the National Optical Astronomy Observatory, Tucson, Arizona 85719, USA.

e-mail:nar@noao.edu

1. Bouwens, R. J. et al. Nature 469, 504-507 (2011).

2. Kimble, R. A. et al. Proc. SPIE 7010, 1-12 (2008).

3. Steidel, C. C. \& Hamilton, D. Astron. J. 104, 941-949 (1992).

4. Bouwens, R. J. et al. Astrophys. J. 686, 230-250 (2008).

\title{
Why are whales big?
}

\section{Different groups of diving vertebrates vary greatly in size, with whales being by far the largest. A comparative investigation of the links between swimming speed, size and metabolism provides clues to the reasons.}

\section{GRAEME D. RUXTON}

A nimals that breathe air but forage under water are highly adapted to the challenges of their extreme lifestyle. The oxygen collected at the surface must be husbanded carefully under water to maximize foraging efficiency. Because the energetic cost of swimming increases rapidly with speed, such divers have long been predicted to ascend and descend at the speed that minimizes the energetic cost (and thus the oxygen burned in aerobic metabolism) for the distance travelled.

In a paper in Journal of Animal Ecology, Watanabe et al. ${ }^{1}$ present the strongest evidence yet in support of such fine-tuned adaptation. Key to their study is the consideration of how we might expect both size and metabolic rate to influence a diver's swimming speed. On the basis of established biomechanical and energetic principles ${ }^{2}$, the authors predicted that larger divers should swim faster (specifically, that swimming speed should increase with mass to the power 0.05 ). They also predicted that low-metabolism ectotherms (animals, such as turtles, whose temperature is strongly influenced by their environment) should swim more slowly than same-sized, highmetabolism endotherms (which maintain a constant temperature; for example, birds and mammals).

Telemetry studies of free-living animals are becoming increasingly common, making it possible for the researchers ${ }^{1}$ to assemble dive-speed data for 37 species encompassing mammals, birds and turtles, and ranging in size from a 500-gram rhinoceros auklet (Cerorhinca monocerata) to a 90-tonne blue whale (Balaenoptera musculus). They found that dive speed does indeed increase with size, and that the mass exponent of the increase (mean $0.09,95 \%$ confidence interval $0.04-0.14$ ) provided a good match to their theoretical predictions. They also found that the three turtle species in the data set all had slower swimming speeds than expected on the basis of their mass: just as the authors' theory predicted for ectotherms.

Although fish are the oldest and most diverse group of diving organisms, they do not reach the gigantic size attained by some other marine divers. The largest-known extant fish is the 6-metre-long whale shark (Rhincodon typus) and the largest extinct fish the 9-metrelong Leedsichthys ${ }^{3}$. Compare these species with the two groups of whales: baleen and toothed. Among the 15 extant baleen whales, only the pygmy right whale (Caperea marginata) is as small as the whale shark, and only this species and the common minke (Balaenoptera acutorostrata) are smaller than Leedsichthys. Among the toothed whales, meanwhile, there are at least five species larger than any extant fish. Turning to extinct marine reptiles ${ }^{4}$, mosasaurs ranged up to $17 \mathrm{~m}$ in length, pliosaurs and plesiosaurs were at least $15 \mathrm{~m}$ and $20 \mathrm{~m}$, respectively, and ichthyosaurs were perhaps as large as $21 \mathrm{~m}$.

From these figures, then, explanation is required for the different maximum sizes of the three groups: ectotherms that draw their oxygen from the water (fish); air-breathing endothermic divers (whales); and air-breathing ectothermic divers, both extant and extinct (turtles and species such as mosasaurs). That explanation might run as follows. First, larger divers can stay submerged for longer because oxygen stores increase more rapidly with size than do metabolic rate or the cost of swimming ${ }^{5}$. Second, as Watanabe et al. ${ }^{1}$ demonstrate, larger divers swim faster.

Taken together, these considerations mean that larger breath-holding divers can exploit deeper waters and search for food more efficiently. However, these selection pressures do not act on fish size. The effect of size on swimming speed is less drastic in modernday turtles, probably because their ectothermic metabolism constrains swimming speed. Thus the prediction is that ectothermic breathholding divers should still face selection for large size, but that this selection will not be as strong as in endotherms such as whales. In agreement with this prediction, the largest extinct marine reptiles were larger than any fish, but not as large as the biggest whales.

This is a thought-provoking study ${ }^{1}$, and there are clear ways in which it could be built upon. Further data on diving ectotherms would be helpful, along with development of the theory to give quantitative predictions for the influence of metabolism. Particularly valuable data would be those that allowed assessment of variation in both resting metabolic rate and dive speed in a given species of turtle across waters of different temperatures (and thus functioning across a range of metabolic rates).

Perhaps the least satisfying aspect of the current theory is that it cannot explain the trend in the empirical data for avian divers to swim faster than mammals of the same mass. Watanabe et al. ${ }^{1}$ suggest that their assumption that metabolism does not increase to cope with thermoregulation in water may be more valid for mammals than birds. Expansion of the species available for the comparative analyses would help in evaluating such theories: the largest bird considered was the $25-\mathrm{kg}$ Emperor penguin (Aptenodytes forsteri) and the smallest mammal was the $33-\mathrm{kg}$ Antarctic fur seal (Arctocephalus gazella). Measurements of small diving mammals, such as otters, might be particularly instructive for comparison with those of same-sized birds.

A final puzzle is why gigantic species have not evolved in two groups of extant vertebrate divers (birds and turtles). Perhaps the difference between these and the other vertebrate divers is that birds and turtles must return to land to reproduce, and it is this phase of their existence (in which they lack the buoyancy of water to support much of their weight) that limits their sizes.

Clearly, diving animals still pose many fascinating questions. As Watanabe et al. ${ }^{1}$ show, however, modern data-collection technologies, combined with biomechanical modelling and comparative approaches, can bring the answers closer to our reach. $\square$

Graeme D. Ruxton is in the College of Medical, Veterinary and Life Sciences, University of Glasgow, Glasgow G12 8QQ, UK. e-mail:graeme.ruxton@glasgow.ac.uk

1. Watanabe, Y. Y. et al. J. Anim. Ecol. 80, 57-68 (2011)

2. Hansen, E. S. \& Ricklefs, R. E. Am. Nat. 163 , 358-374 (2004).

3. Friedman, M. et al. Science 327, 990-993 (2010)

4. Nicholls, E. L. \& Manabe, M. J. Vert. Paleontol. 24. 838-849 (2004)

5. Halsey, L. G., Butler, P. J. \& Blackburn, T. M. Am. Nat $167,276-287$ (2006) 